Hassan Azad • Indranil Biswas

\title{
Holomorphic connections on holomorphic bundles on Riemann surfaces
}

Received: 30 September 2020 / Accepted: 21 February 2021 / Published online: 19 May 2021

(C) The Author(s) 2021

\begin{abstract}
We investigate aspects of holomorphic connections on holomorphic principal bundles over a Riemann surface.
\end{abstract}

Mathematics Subject Classification $53 \mathrm{~B} 15 \cdot 14 \mathrm{H} 60 \cdot 32 \mathrm{~A} 27$

\section{Introduction}

Holomorphic connections, introduced by Atiyah [2], arise in innumerable contexts in mathematics (see [5,9, $11,14,16]$ and references therein). Their importance can hardly be overemphasized. In this mostly expository work, we consider holomorphic connections, and also logarithmic connections, on holomorphic principal bundles over compact Riemann surfaces.

A famous theorem of Atiyah and Weil says that a holomorphic vector bundle $E$ over a compact Riemann surface $M$ admits a holomorphic connection if and only if the degree of every holomorphic direct summand of $E$ is zero. We recall that in the context of a compact Riemann surface, the degree of a complex vector bundle coincides with its first Chern class; in particular, the degree of a complex vector bundle coincides with the degree of the complex line bundle given by its top exterior product. In [3], this result was extended to holomorphic principal $G$-bundles on $M$, where $G$ is a connected reductive complex algebraic group. More precisely, the following was proved in [3]:

Theorem 1.1 Let $G$ be a connected reductive affine algebraic group defined over $\mathbb{C}$. A holomorphic principal $G$-bundle $E_{G}$ over a compact connected Riemann surface $M$ admits a holomorphic connection if and only if for every triple of the form $\left(H, E_{H}, \lambda\right)$, where

(1) $H$ is a Levi subgroup of $G$,

(2) $E_{H} \subset E_{G}$ is a holomorphic reduction of structure group to $H$, and

(3) $\lambda$ is a holomorphic character of $H$,

the associated line bundle $E_{H}(\lambda)=\left(E_{H} \times \mathbb{C}\right) / H$ over $M$ is of degree zero.

H. Azad

Abdus Salam School of Mathematical Sciences, GC University Lahore, 68-B, New Muslim Town, Lahore 54600, Pakistan

E-mail: hassan.azad@sms.edu.pk

I. Biswas $(\varangle)$

School of Mathematics, Tata Institute of Fundamental Research, Homi Bhabha Road, Mumbai 400005, India

E-mail: indranil@math.tifr.res.in 
The assumption Theorem 1.1 that $G$ is reductive is rather essential; see Sect. 4.

In the final section, we consider logarithmic connections on a compact Riemann surface. The following theorem was proved in [4]:

Theorem 1.2 Take a holomorphic vector bundle E on M. Fix a finite subset

$$
D:=\left\{x_{1}, \ldots, x_{n}\right\} \subset M .
$$

For each point $x \in D$, fix

$$
T_{x} \in \operatorname{End}\left(E_{x}\right)
$$

such that $\phi(x) \circ T_{x}=T_{x} \circ \phi(x)$ for every holomorphic automorphism $\phi$ of the holomorphic vector bundle $E$. There is a logarithmic connection $\theta$ on E singular over $D$ such that the residue

$$
\operatorname{Res}(\theta, x)=T_{x}
$$

if and only if

$$
\operatorname{degree}(E)+\sum_{x \in D} \operatorname{trace}\left(\left.\operatorname{Res}(\theta, x)\right|_{E_{x}}\right)=0 .
$$

When $D=0$, Theorem 1.2 coincides with the above-mentioned criterion of Atiyah and Weil for the existence of a holomorphic connection on a holomorphic vector bundle over $M$.

\section{Holomorphic connection on principal bundles}

Let $X$ be a compact connected complex manifold. Let $G$ be a complex Lie group. A holomorphic principal $G$-bundle over $X$ consists of

- a complex manifold $E_{G}$,

- a surjective holomorphic map $f: E_{G} \longrightarrow X$, and

- a holomorphic right action $\varphi: E_{G} \times G \longrightarrow E_{G}$ of $G$ on $E_{G}$,

such that the following two conditions hold:

(1) $f \circ \varphi=f \circ p_{1}$, where $p_{1}: E_{G} \times G \longrightarrow E_{G}$ is the natural projection, and

(2) the map to the fiber product

$$
E_{G} \times G \longrightarrow E_{G} \times{ }_{X} E_{G}, \quad(z, g) \longmapsto(z, \varphi(z, g))
$$

is a biholomorphism.

Note that the first condition implies that $(z, \varphi(z, g)) \in E_{G} \times_{X} E_{G}$.

For notational convenience, $\varphi(z, g)$, where $z \in E_{G}$ and $g \in G$, will be denoted by $z g$.

The holomorphic tangent (respectively, cotangent) bundle of a complex manifold $Y$ will be denoted by $T Y$ (respectively, $\Omega_{Y}^{1}$ ).

The Lie algebra of $G$ will be denoted by $\mathfrak{g}$. Let

$$
f: E_{G} \longrightarrow X
$$

be a holomorphic principal $G$-bundle over $X$. Consider the holomorphic right action of $G$ on the holomorphic tangent bundle $T E_{G}$ induced by the right action of $G$ on $E$. The quotient

$$
\operatorname{At}\left(E_{G}\right):=\left(T E_{G}\right) / G
$$

is a complex manifold; in fact, it is a holomorphic vector bundle over $E_{G} / G=X$. This holomorphic vector bundle $\operatorname{At}\left(E_{G}\right)$ over $X$ is called the Atiyah bundle for $E_{G}$ [2]. The differential

$$
d f: T E_{G} \longrightarrow f^{*} T X
$$


of the projection $f$ is evidently $G$-equivariant for the natural action of $G$ on $f^{*} T X$ that lifts the action of $G$ on $E_{G}$. The action of $G$ on $E_{G}$ produces a holomorphic homomorphism from the trivial holomorphic bundle $E_{G} \times \mathfrak{g} \longrightarrow E_{G}$ with fiber $\mathfrak{g}$

$$
E_{G} \times \mathfrak{g} \longrightarrow \operatorname{kernel}(d f)
$$

which is in fact an isomorphism. From this it follows that we have a short exact sequence of holomorphic vector bundles on $E_{G}$

$$
0 \longrightarrow \operatorname{kernel}(d f)=E_{G} \times \mathfrak{g} \longrightarrow T E_{G} \stackrel{d f}{\longrightarrow} f^{*} T X \longrightarrow 0 ;
$$

all the homomorphisms in (2.3) are $G$-equivariant. The quotient kernel $(d f) / G$ is called the adjoint vector bundle, and it is denoted by $\operatorname{ad}\left(E_{G}\right)$. This holomorphic vector bundle $\operatorname{ad}\left(E_{G}\right)$ over $X$ is identified with the holomorphic vector bundle $E_{G}(\mathfrak{g})$ associated to $E_{G}$ for the adjoint action of $G$ on the Lie algebra $\mathfrak{g}$; this identification is produced by the isomorphism in (2.2).

Taking quotient of the holomorphic vector bundles in (2.3), by the actions of $G$, the following short exact sequence of holomorphic vector bundles on $X$ is obtained:

$$
0 \longrightarrow \operatorname{ad}\left(E_{G}\right) \longrightarrow \operatorname{At}\left(E_{G}\right) \stackrel{d^{\prime} f}{\longrightarrow} T X \longrightarrow 0,
$$

where $d^{\prime} f$ is the descent of the homomorphism $d f$ (see [2]). The short exact sequence in (2.4) is known as the Atiyah exact sequence for $E_{G}$.

A holomorphic connection on $E_{G}$ is a holomorphic homomorphism of vector bundles

$$
\mathcal{D}: T X \longrightarrow \operatorname{At}\left(E_{G}\right)
$$

such that

$$
\left(d^{\prime} f\right) \circ \mathcal{D}=\operatorname{Id}_{T X},
$$

where $d^{\prime} f$ is the projection in (2.4) (see [2]). Therefore, $E_{G}$ admits a holomorphic connection if and only if the Atiyah exact sequence in (2.4) splits holomorphically.

Giving a holomorphic connection on $E_{G}$ is equivalent to giving a $\mathfrak{g}$-valued holomorphic 1-form

$$
\omega \in H^{0}\left(E_{G}, \Omega_{E_{G}}^{1} \otimes_{\mathbb{C}} \mathfrak{g}\right)
$$

on $E_{G}$ such that the following two conditions hold:

- the homomorphism

$$
\omega: T E_{G} \longrightarrow \mathfrak{g}
$$

is $G$-equivariant for the adjoint action of $G$ on $\mathfrak{g}$ and the action of $G$ on $T E_{G}$ given by the right action of $G$ on $E_{G}$, and

- the restriction of $\omega$ to any fiber of $f$ coincides with the Maurer-Cartan form, equivalently, the restriction of $\omega$ to any fiber of $f$ coincides with the inverse of the isomorphism in (2.2).

For a form $\omega$ as in (2.5) giving a holomorphic connection on $E_{G}$, let

$$
\operatorname{kernel}(\omega) \subset T E_{G}
$$

be the kernel of the homomorphism $T E_{G} \longrightarrow E_{G} \times \mathfrak{g}$ given by $\omega$. The action of $G$ on $T E_{G}$ preserves this holomorphic subbundle $\operatorname{kernel}(\omega)$. Consequently,

$$
\operatorname{kernel}(\omega) / G \subset\left(T E_{G}\right) / G=: \operatorname{At}\left(E_{G}\right)
$$

is a holomorphic subbundle. There is a unique holomorphic homomorphism

$$
\mathcal{D}: T X \longrightarrow \operatorname{At}\left(E_{G}\right)
$$

such that 
- $\mathcal{D}(T X) \subset \operatorname{kernel}(\omega) / G$, and

- $\left(d^{\prime} f\right) \circ \mathcal{D}=\operatorname{Id}_{T X}$, where $d^{\prime} f$ is the homomorphism in (2.4).

Therefore, $\mathcal{D}$ defines a holomorphic connection on $E_{G}$.

Conversely, given a holomorphic homomorphism

$$
\mathcal{D}: T X \longrightarrow \operatorname{At}\left(E_{G}\right)
$$

such that $\left(d^{\prime} f\right) \circ \mathcal{D}=\operatorname{Id}_{T X}$, let

$$
V \subset T E_{G}
$$

be the inverse image of $\mathcal{D}(T X) \subset \operatorname{At}\left(E_{G}\right)$ under the quotient map

$$
T E_{G} \longrightarrow\left(T E_{G}\right) / G=: \operatorname{At}\left(E_{G}\right) .
$$

Then there is a unique holomorphic 1-form $\omega \in H^{0}\left(E_{G}, \Omega_{E_{G}}^{1} \otimes \mathbb{C} \mathfrak{g}\right)$ such that

- the kernel of the homomorphism $T E_{G} \longrightarrow E_{G} \times \mathfrak{g}$ given by $\omega$ coincides with the subbundle $V \subset T E_{G}$ in (2.6), and

- the restriction of $\omega$ to any fiber of $f$ coincides with the Maurer-Cartan form.

The above homomorphism $\omega: T E_{G} \longrightarrow \mathfrak{g}$ is $G$-equivariant, and hence we conclude that $\omega$ defines a holomorphic connection on $E_{G}$.

For a complex manifold $Z$, the Lie bracket operation of locally defined holomorphic vector fields on $Z$ makes the coherent analytic sheaf $T Z$ a sheaf of Lie algebras. Note that the Lie bracket operation on locally defined holomorphic vector fields on $Z$ is not $\mathcal{O}_{Z}$-linear.

The Lie bracket of two $G$-invariant vector fields on $\left.E_{G}\right|_{U}$, where $U \subset X$ is an open subset, is again $G$-invariant. Consequently, the Lie algebra structure on the coherent analytic sheaf $T E_{G}$ makes the coherent analytic sheaf $\operatorname{At}\left(E_{G}\right)$ a sheaf of Lie algebras. Take a holomorphic connection

$$
\mathcal{D}: T X \longrightarrow \operatorname{At}(E)
$$

on $E_{G}$. The curvature of $\mathcal{D}$ is the obstruction for the homomorphism $\mathcal{D}$ to be compatible with the Lie algebra structures on the sheaves $\operatorname{At}\left(E_{G}\right)$ and $T X$. More precisely, given holomorphic vector fields $s, t$ defined on an open subset $U \subset X$, consider

$$
\mathcal{K}(\mathcal{D})(s, t):=[\mathcal{D}(s), \mathcal{D}(t)]-\mathcal{D}([s, t]) \in H^{0}\left(U, \operatorname{At}\left(E_{G}\right)\right) .
$$

Since the projection $d^{\prime} f$ in (2.4) is Lie algebra structure preserving, it follows that

$$
\mathcal{K}(\mathcal{D})(s, t) \in H^{0}\left(U, \operatorname{ad}\left(E_{G}\right)\right) .
$$

Furthermore, we have

$$
\mathcal{K}(\mathcal{D})(s, t)=-\mathcal{K}(\mathcal{D})(t, s)
$$

and

$$
\mathcal{K}(\mathcal{D})(\phi \cdot s, t)=-\phi \cdot \mathcal{K}(\mathcal{D})(t, s)
$$

for all $\phi \in H^{0}\left(U, \mathcal{O}_{U}\right)$. These properties together imply that

$$
\mathcal{K}(\mathcal{D}) \in H^{0}\left(X, \operatorname{ad}\left(E_{G}\right) \otimes \Omega_{X}^{2}\right) .
$$

This $\operatorname{ad}\left(E_{G}\right)$-valued holomorphic 2-form $\mathcal{K}(\mathcal{D})$ on $X$ is the curvature of $D$.

The connection $D$ is called flat (or integrable) if $\mathcal{K}(\mathcal{D})=0$. A connection $D$ is flat if and only if the distribution on $E_{G}$ given by the subbundle $V \subset T E_{G}$ in (2.6) corresponding to $\mathcal{D}$ is integrable [15, p. 78, Corollary 5.3], [10].

If $\operatorname{dim}_{\mathbb{C}} X=1$, then $\Omega_{X}^{2}=0$. Therefore, any holomorphic connection on a holomorphic principal $G$-bundle over a Riemann surface is automatically flat. 


\section{Holomorphic connections on bundles over a Riemann surface}

In this section we will consider holomorphic principal $G$-bundles on compact Riemann surfaces and holomorphic connections on them.

Let $M$ be a compact connected Riemann surface. As before, $G$ is a complex Lie group. Given a holomorphic principal $G$-bundle $E_{G}$ on $M$, it is natural to ask whether $E_{G}$ admits a holomorphic connection. A well-known theorem of Atiyah and Weil answers this question when $G=\operatorname{GL}(r, \mathbb{C})[2,17]$. This result of [2,17] will be recalled below.

Using the standard representation of $\mathrm{GL}(r, \mathbb{C})$, holomorphic principal $\mathrm{GL}(r, \mathbb{C})$-bundles on $M$ are identified with holomorphic vector bundles on $M$ of rank $r$.

A holomorphic vector bundle $W$ on a compact connected complex manifold $X$ is called decomposable if

$$
W=W_{1} \oplus W_{2},
$$

where $W_{1}$ and $W_{2}$ are holomorphic vector bundles of positive rank. A holomorphic vector bundle $W$ on $X$ is called indecomposable if it is not decomposable. Every holomorphic vector bundle on $X$ is holomorphically isomorphic to a direct sum of indecomposable holomorphic vector bundles.

The following very important theorem was proved by Atiyah.

Theorem 3.1 ([1, p. 315, Theorem 3]) Let $W$ be a holomorphic vector bundle over a compact connected complex manifold $X$. If $W$ is holomorphically isomorphic to both $\bigoplus_{i=1}^{m} V_{i}$ and $\bigoplus_{j=1}^{n} W_{j}$, where $V_{i}, 1 \leq i \leq$ $m$, and $W_{j}, 1 \leq j \leq n$, are all indecomposable vector bundles on $X$, then

(1) $m=n$, and

(2) there is a permutation $\sigma$ of $\{1, \ldots, m\}$ such that $V_{i}$ is holomorphically isomorphic to $W_{\sigma(i)}$ for all $1 \leq i \leq m$.

The above-mentioned theorem of $[2,17]$ says that a holomorphic vector bundle $E$ over a compact connected Riemann surface $M$ admits a holomorphic connection if and only if each direct summand of $E$ is of degree zero. Let

$$
E=\bigoplus_{i=1}^{m} V_{i}
$$

be a decomposition of $E$ into a direct sum of indecomposable vector bundles (as in Theorem 3.1). Then $E$ admits a holomorphic connection if and only if

$$
\operatorname{degree}\left(V_{i}\right)=0
$$

for all $1 \leq i \leq m$.

We shall now describe a generalization of the above theorem of [2] and [17] to the context of principal bundles.

Let $G$ be a connected reductive affine algebraic group defined over $\mathbb{C}$. A Zariski closed connected subgroup $P \subset G$ is called a parabolic subgroup if $G / P$ is a projective variety $[8,11.2]$, [13]. The unipotent radical of a parabolic subgroup $P \subset G$ will be denoted by $R_{u}(P)$. The quotient group $P / R_{u}(P)$ is called the Levi quotient of $P$. A Levi factor of $P$ is a Zariski closed connected subgroup $L \subset P$ such that the composition

$$
L \hookrightarrow P \longrightarrow P / R_{u}(P)
$$

is an isomorphism [13, p. 184]. We note that $P$ admits Levi factors, and any two Levi factors of $P$ are conjugate by an element of $R_{u}(P)[13, \S 30.2$, p. 185, Theorem]. A Levi factor $L \subset P$ satisfies the following condition: $L$ contains a maximal torus of $P$, and moreover $L$ is a maximal reductive subgroup of $P$ (see $[8,13]$ ).

Given a holomorphic principal $G$-bundle $E_{G}$ on $M$ and a complex Lie subgroup $H \subset G$, a holomorphic reduction of $E_{G}$ to $H$ is given by a holomorphic section of the holomorphic fiber bundle $E_{G} / H$ over $M$. Let

$$
q_{H}: E_{G} \longrightarrow E_{G} / H
$$

be the quotient map. If $v: M \longrightarrow E_{G} / H$ is a holomorphic section of the fiber bundle $E_{G} / H \longrightarrow M$, then note that

$$
q_{H}^{-1}(v(M)) \subset E_{G}
$$


is a holomorphic principal $H$-bundle on $M$. Conversely, if a complex submanifold $E_{H} \subset E_{G}$ is preserved by the action of $H$ on $E_{G}$, and $E_{H}$ is a principal $H$-bundle for the action of $H$, then the natural map

$$
M=E_{H} / H \hookrightarrow E_{G} / H
$$

is a holomorphic section of the projection $E_{G} / H \longrightarrow M$.

If $E_{H}$ is a holomorphic principal $H$-bundle on $M$, and $\chi$ is a holomorphic character of $H$, then the associated holomorphic line bundle

$$
E_{H}(\lambda)=\left(E_{H} \times \mathbb{C}\right) / H \longrightarrow M
$$

is the quotient of $E_{H} \times \mathbb{C}$, where $\left(z_{1}, c_{1}\right),\left(z_{2}, c_{2}\right) \in E_{H} \times \mathbb{C}$ are identified if there is an element $g \in H$ such that

- $z_{2}=z_{1} g$, and

- $c_{2}=\frac{c_{1}}{\lambda(g)}$.

The following theorem is proved in [3] (see [3, Theorem 4.1]).

Theorem 3.2 Let $G$ be a connected reductive affine algebraic group defined over $\mathbb{C}$. A holomorphic principal $G$-bundle $E_{G}$ over a compact connected Riemann surface $M$ admits a holomorphic connection if and only if for every triple of the form $\left(H, E_{H}, \lambda\right)$, where

(1) $H$ is a Levi subgroup of $G$,

(2) $E_{H} \subset E_{G}$ is a holomorphic reduction of structure group to $H$, and

(3) $\lambda$ is a holomorphic character of $H$,

the associated line bundle $E_{H}(\lambda)=\left(E_{H} \times \mathbb{C}\right) / H$ (see (3.1)) over $M$ is of degree zero.

Note that if we set $G=\operatorname{GL}(n, \mathbb{C})$ in Theorem 3.2, then it coincides with the mentioned criterion of Atiyah and Weil for the existence of a holomorphic connection on a holomorphic vector bundle over $M$.

A sketch of the proof of Theorem 3.2 will be given below.

Let $E_{G}$ be a holomorphic principal $G$-bundle over $M$ equipped with a holomorphic connection $\nabla$. Take any triple $\left(H, E_{H}, \lambda\right)$ as in Theorem 3.2. We will first show that a holomorphic connection $\nabla$ on $E_{G}$ produces a holomorphic connection on the principal $H$-bundle $E_{H}$.

Let $\mathfrak{g}$ and $\mathfrak{h}$ denote the Lie algebras of $G$ and $H$ respectively. The group $H$ has adjoint actions on both $\mathfrak{h}$ and $\mathfrak{g}$. To construct the connection on $E_{H}$, fix a splitting of the injective homomorphism of $H$-modules

$$
0 \longrightarrow \mathfrak{h} \longrightarrow \mathfrak{g}
$$

Let

$$
\rho: \mathfrak{g} \longrightarrow \mathfrak{h}
$$

be the chosen splitting. Since a holomorphic connection on $E_{G}$ is a given by a holomorphic splitting of the Atiyah exact sequence for $E_{G}$, a holomorphic connection $\nabla$ on $E_{G}$ produces a $\mathfrak{g}$-valued holomorphic 1-form $\omega$ on $E_{G}$ satisfying the following two conditions:

- $\omega$ is $G$-equivariant ( $G$ acts on $\mathfrak{g}$ by inner automorphism), and

- the restriction of $\omega$ to any fiber of $E_{G}$ is the Maurer-Cartan form on the fiber.

Using the chosen splitting homomorphism

$$
\mathfrak{g} \stackrel{\rho}{\longrightarrow} \mathfrak{h},
$$

the connection form $\omega$ on $E_{G}$ defines a $\mathfrak{h}$-valued holomorphic one-form $\omega^{\prime}$ on $E_{G}$. The restriction of $\omega^{\prime}$ to the complex submanifold $E_{H} \subset E_{G}$ satisfies the two conditions needed for a holomorphic $\mathfrak{h}$-valued 1-form on $E_{H}$ to define a holomorphic connection on $E_{H}$.

Therefore, $E_{H}$ admits a holomorphic connection. A holomorphic connection on $E_{H}$ induces a holomorphic connection on the associated line bundle $E_{H}(\lambda)$. Any line bundle admitting a holomorphic connection must be of degree zero [2]. Therefore, if $E_{G}$ admits a holomorphic connection then we know that the degree of $E_{H}(\lambda)$ is zero. 
To prove the converse, let $E_{G}$ be a holomorphic principal $G$-bundle over $M$ such that

$$
\operatorname{degree}\left(E_{H}(\lambda)\right)=0
$$

for all triples $\left(H, E_{H}, \lambda\right)$ of the above type. We need to show that the Atiyah exact sequence for $E_{G}$ (see (2.4)) splits holomorphically.

As the first step for it, in [3] the following is proved: it is enough to prove that the Atiyah exact sequence for $E_{G}$ splits holomorphically under the assumption that $E_{G}$ does not admit any holomorphic reduction of structure group to any proper Levi subgroup of $G$. Therefore, we assume that $E_{G}$ does not admit any holomorphic reduction of structure group to any proper Levi subgroup of $G$.

Let $K_{M}$ denote the holomorphic cotangent bundle of $M$. The obstruction for splitting of the Atiyah exact sequence for $E_{G}$ is an element

$$
\tau\left(E_{G}\right) \in H^{1}\left(M, K_{M} \otimes \operatorname{ad}\left(E_{G}\right)\right) .
$$

We note that

$$
\operatorname{ad}\left(E_{G}\right)^{*}=\operatorname{ad}\left(E_{G}\right) .
$$

Indeed, the Lie algebra $\mathfrak{g}$ admits a $G$-invariant symmetric nondegenerate bilinear form. Such a form on $\mathfrak{g}$ produces an isomorphism of $\operatorname{ad}\left(E_{G}\right)$ with $\operatorname{ad}\left(E_{G}\right)^{*}$. Consequently, by Serre duality,

$$
H^{1}\left(M, K_{M} \otimes \operatorname{ad}\left(E_{G}\right)\right)=H^{0}\left(M, \operatorname{ad}\left(E_{G}\right)\right)^{*} .
$$

So we have

$$
\tau\left(E_{G}\right) \in H^{0}\left(M, \operatorname{ad}\left(E_{G}\right)\right)^{*} .
$$

Any homomorphic section $\phi$ of the adjoint bundle $\operatorname{ad}\left(E_{G}\right)$ has a Jordan decomposition

$$
\phi=f_{s}+f_{n},
$$

where $f_{s}$ is pointwise semisimple and $f_{n}$ is pointwise nilpotent. From the assumption that $E_{G}$ does not admit any holomorphic reduction of structure group to any proper Levi subgroup of $G$ it can be deduced that the semisimple section $f_{s}$ is given by some element of the center of $\mathfrak{g}$. Indeed, the conjugacy class of $f_{s}(x)$ is independent of $x \in M$; this follows from the fact that there is no nonconstant holomorphic map from $M$ to the affine variety that parametrizes the conjugacy classes of semisimple elements in $\mathfrak{g}$. Fix an element $z_{0} \in \mathfrak{g}$ in the conjugacy class determined by $f_{s}(x)$. Then the centralizer of $z_{0}$ in $G$ is a Levi subgroup $L\left(z_{0}\right)$ of some parabolic subgroup of $G$. Note that for any $y \in E_{G}$ and $z^{\prime} \in \mathfrak{g}$, the pair $\left(y, z^{\prime}\right)$ represents an element of $\operatorname{ad}\left(E_{G}\right)$. It is straightforward to check that

$$
E_{L\left(z_{0}\right)}:=\left\{y \in E_{G} \mid\left(y, z_{0}\right) \in f_{s}(M)\right\} \subset E_{G}
$$

is a holomorphic reduction of structure group of $E_{G}$ to the subgroup $L\left(z_{0}\right)$ of $G$. Now from the given assumption that $E_{G}$ does not admit any holomorphic reduction of structure group to any proper Levi subgroup of $G$ we conclude that $L\left(z_{0}\right)=G$. Consequently, the semisimple section $f_{s}$ is given by some element of the center of $\mathfrak{g}$.

Since the semisimple section $f_{s}$ is given by some element of the center of $\mathfrak{g}$, from the given condition on $E_{G}$ it can be deduced that

$$
\tau\left(E_{G}\right)\left(f_{s}\right)=0,
$$

where $\tau\left(E_{G}\right)$ is the element in (3.3).

The nilpotent section $f_{n}$ of ad $\left(E_{G}\right)$ gives a holomorphic reduction of structure group

$$
E_{P} \subset E_{G}
$$

of $E_{G}$ to a proper parabolic subgroup $P$ of $G$. This reduction $E_{P}$ has the property that $f_{n}$ lies in the image

$$
H^{0}\left(M, \mathcal{R}\left(\operatorname{ad}\left(E_{P}\right)\right)\right) \hookrightarrow H^{0}\left(M, \operatorname{ad}\left(E_{P}\right)\right) \hookrightarrow H^{0}\left(M, \operatorname{ad}\left(E_{G}\right)\right),
$$


where $\operatorname{ad}\left(E_{P}\right)$ is the adjoint bundle of $E_{P}$, and

$$
\mathcal{R}\left(\operatorname{ad}\left(E_{P}\right)\right) \subset \operatorname{ad}\left(E_{P}\right)
$$

is the nilpotent radical bundle of $\operatorname{ad}\left(E_{P}\right)$. On the other hand, the cohomology class $\tau\left(E_{G}\right) \in H^{1}\left(M, K_{M} \otimes\right.$ $\left.\operatorname{ad}\left(E_{G}\right)\right)$ in (3.2) lies in the image of the homomorphism

$$
H^{1}\left(M, K_{M} \otimes \operatorname{ad}\left(E_{P}\right)\right) \longrightarrow H^{1}\left(M, K_{M} \otimes \operatorname{ad}\left(E_{G}\right)\right)
$$

induced by the inclusion map $\operatorname{ad}\left(E_{P}\right) \hookrightarrow \operatorname{ad}\left(E_{G}\right)$.

next we observe the following. Let $\mathfrak{p}$ be the Lie algebra of $P$, and let $\mathcal{R}(\mathfrak{p}) \subset \mathfrak{p}$ be the nilpotent radical of $\mathfrak{p}$. Then for any $G$-invariant symmetric bilinear form $B$ on $\mathfrak{g}$, we have

$$
B(\mathfrak{p}, \mathcal{R}(\mathfrak{p})=0 .
$$

This, and the above observations that

- $f_{n}$ lies in the image

$$
H^{0}\left(M, \mathcal{R}\left(\operatorname{ad}\left(E_{P}\right)\right)\right) \hookrightarrow H^{0}\left(M, \operatorname{ad}\left(E_{G}\right)\right)
$$

and

- $\tau\left(E_{G}\right) \in H^{1}\left(M, K_{M} \otimes \operatorname{ad}\left(E_{G}\right)\right)$ lies in the image of the homomorphism

$$
H^{1}\left(M, K_{M} \otimes \operatorname{ad}\left(E_{P}\right)\right) \longrightarrow H^{1}\left(M, K_{M} \otimes \operatorname{ad}\left(E_{G}\right)\right),
$$

combine together to imply that $\tau\left(E_{G}\right)\left(f_{n}\right)=0$.

Hence $\tau\left(E_{G}\right)(\phi)=0$ for all $\phi \in H^{0}\left(M, \operatorname{ad}\left(E_{G}\right)\right)$, which implies that $\tau\left(E_{G}\right)=0$. Therefore, the Atiyah exact sequence for $E_{G}$ splits holomorphically, implying that the holomorphic principal $G$-bundle $E_{G}$ admits a holomorphic connection.

\subsection{Non-reductive algebraic groups}

Now let $G$ be a unipotent affine algebraic group defined over $\mathbb{C}$. It is know that any holomorphic principal $G$ bundle over a compact connected Riemann surface $M$ admits a holomorphic connection [7, p. 4015, Corollary 3.8].

Let $G$ be a connected affine algebraic group defined over $\mathbb{C}$. The unipotent radical of $G$ will be denoted by $R_{u}(G)$. Let

$$
L(G):=G / R_{u}(G)
$$

be the reductive quotient. Let $E_{G}$ be a holomorphic principal $G$-bundle over a compact connected Riemann surface $M$. Assume that the holomorphic principal $L(G)$-bundle $E_{G} / R_{u}(G)$ admits a holomorphic connection. The following is a natural question to ask:

Does the holomorphic principal G-bundle $E_{G}$ admit a holomorphic connection?

We will show in Sect. 4 that the above question has a negative answer.

\section{A construction}

This section is an exposition of [6].

Let $M$ be a compact connected Riemann surface of genus $g$, with $g \geq 2$. Denote by $K_{M}$ the holomorphic cotangent bundle of $M$. The linear equivalence class of $K_{M}$ can be expressed as

$$
K_{M}=P+D,
$$

where $P \in M$ is a single point and $D$ is an effective divisor of degree $2 g-3$ such that $P$ is disjoint from the support of $D$. We note that this can be deduced from the fact that the linear system $K_{M}$ is globally generated and

$$
\operatorname{dim} H^{0}\left(M, K_{M}\right)=g \geq 2 .
$$


Indeed, let

$$
f: M \longrightarrow \mathbb{P}\left(H^{0}\left(M, K_{M}\right)\right)
$$

be the map corresponding to $K_{M}$. Take a hyperplane $\mathbb{H} \subset \mathbb{P}\left(H^{0}\left(M, K_{M}\right)\right)$ such that there is a point $z \in$ $\mathbb{H} \bigcap f(M)$ satisfying the following two conditions:

(1) $\mathbb{H}$ and $f(M)$ intersects transversally at $z$, and

(2) $f$ is unramified over $z$.

Now take $P \in f^{-1}(z)$ and $D=f^{-1}(\mathbb{H}) \backslash\{P\}$.

Fix $P$ and $D$ as in (4.1). Let us now split the divisor $D$ into two parts, namely

$$
D=D_{Q}+D_{R},
$$

where $D_{Q}$ and $D_{R}$ are effective divisors with

$$
\operatorname{degree}\left(D_{Q}\right)+1=\operatorname{degree}\left(D_{R}\right)=g-1 .
$$

By Serre duality,

$$
H^{1}\left(M, \mathcal{O}_{M}\left(D_{Q}+D_{R}\right)\right)=H^{0}\left(M, \mathcal{O}_{M}(P)\right)^{*}=\mathbb{C} .
$$

In particular, we can choose a nonzero element (which is actually unique up to multiplication by a scalar)

$$
\theta \in H^{1}\left(M, \mathcal{O}_{M}\left(D_{Q}+D_{R}\right)\right) \backslash\{0\} .
$$

Since

$$
\mathcal{O}_{M}\left(D_{Q}+D_{R}\right)=\operatorname{Hom}_{\mathcal{O}_{M}}\left(\mathcal{O}_{M}\left(-D_{R}\right), \mathcal{O}_{M}\left(D_{Q}\right)\right)
$$

the cohomology class $\theta$ in (4.5) produces a short exact sequence of holomorphic vector bundles

$$
0 \longrightarrow \mathcal{O}_{M}\left(D_{Q}\right) \longrightarrow V \longrightarrow \mathcal{O}_{M}\left(-D_{R}\right) \longrightarrow 0
$$

on $M$. This exact sequence does not split holomorphically because $\theta \neq 0$. From (4.3), it follows that degree $(V)=-1$. Consider the holomorphic vector bundle

$$
E:=\mathcal{O}_{M}(P) \oplus V
$$

on $M$ of rank three and degree zero. We have

$$
\mathcal{O}_{M}(P) \oplus \mathcal{O}_{M}\left(D_{Q}\right) \subset E
$$

because $\mathcal{O}_{M}\left(D_{Q}\right) \subset V$ (see (4.6)). Let $s^{P}$ (respectively, $s^{Q}$ ) be the holomorphic section of $\mathcal{O}_{M}(P)$ (respectively, $\mathcal{O}_{M}\left(D_{Q}\right)$ ) given by the constant function 1 on $M$. So $s^{P}$ (respectively, $s^{Q}$ ) vanishes over $P$ (respectively, the support of $D_{Q}$ ) of order one, and is nonzero everywhere else. Now consider the holomorphic section

$$
\sigma_{1}: \mathcal{O}_{M} \longrightarrow \mathcal{O}_{M}(P) \oplus \mathcal{O}_{M}\left(D_{Q}\right)
$$

defined by $x \longmapsto\left(s^{P}(x), s^{Q}(x)\right)$. Note that $\sigma_{1}$ does not vanish anywhere because $P$ is disjoint from the support of $D_{Q}$ according to (4.1) and (4.2). Let $\sigma$ be the composition

$$
\mathcal{O}_{M} \stackrel{\sigma_{1}}{\longrightarrow} \mathcal{O}_{M}(P) \oplus \mathcal{O}_{M}\left(D_{Q}\right) \hookrightarrow E
$$

(see (4.8)). Since $\sigma$ is nowhere vanishing, we get a short exact sequence of holomorphic vector bundles on $M$

$$
0 \longrightarrow F:=\mathcal{O}_{M} \stackrel{\sigma}{\longrightarrow} E \longrightarrow \mathcal{Q}:=E / \sigma(F) \longrightarrow 0 .
$$

By construction, $\mathcal{O}_{M}(P)$ is a direct summand of $E$. Since degree $\left(\mathcal{O}_{M}(P)\right) \neq 0$, from the criterion of Atiyah-Weil we conclude that $E$ does not admit a holomorphic connection.

We will now prove that both $F$ and $\mathcal{Q}$ in (4.11) admit a holomorphic connection.

Of course, $F:=\mathcal{O}_{M}$ admits the trivial holomorphic connection. So we need to show that $\mathcal{Q}$ in (4.11) admits a holomorphic connection. The following lemma would be needed for that. 
Lemma 4.1 The holomorphic vector bundle $\mathcal{Q}$ in (4.11) is a nontrivial extension of the line bundle $\mathcal{O}_{M}\left(-D_{R}\right)$ by $\mathcal{O}_{M}\left(P+D_{Q}\right)$.

Proof On the one hand, we have

$$
\bigwedge^{2}\left(\mathcal{O}_{M}(P) \oplus \mathcal{O}_{M}\left(D_{Q}\right)\right)=\mathcal{O}_{M}\left(P+D_{Q}\right)
$$

On the other hand,

$$
\bigwedge^{2}\left(\mathcal{O}_{M}(P) \oplus \mathcal{O}_{M}\left(D_{Q}\right)\right)=\left(\mathcal{O}_{M}(P) \oplus \mathcal{O}_{M}\left(D_{Q}\right)\right) / \sigma_{1}\left(\mathcal{O}_{M}\right)
$$

(see (4.9)). It follows that

$$
\left(\mathcal{O}_{M}(P) \oplus \mathcal{O}_{M}\left(D_{Q}\right)\right) / \sigma_{1}\left(\mathcal{O}_{M}\right)=\mathcal{O}_{M}\left(P+D_{Q}\right)
$$

The inclusion of $\mathcal{O}_{M}(P) \oplus \mathcal{O}_{M}\left(D_{Q}\right)$ in $E$ (see (4.10)) produces an inclusion of the quotient $\left(\mathcal{O}_{M}(P) \oplus\right.$ $\left.\mathcal{O}_{M}\left(D_{Q}\right)\right) / \sigma_{1}\left(\mathcal{O}_{M}\right)$ in $E / \sigma(F)=\mathcal{Q}$ (see (4.11)). Therefore, from (4.12), we have

$$
\mathcal{O}_{M}\left(P+D_{Q}\right) \subset \mathcal{Q}
$$

as a subbundle. Using (4.6), (4.7) we have

$$
\bigwedge^{2} \mathcal{Q}=\bigwedge^{3} E=\mathcal{O}_{M}(P) \otimes \bigwedge^{2} V=\mathcal{O}_{M}\left(P+D_{Q}-D_{R}\right)
$$

Note that its degree is zero (4.3). Therefore, from (4.13),

$$
\mathcal{O}_{M}\left(P+D_{Q}-D_{R}\right)=\bigwedge^{2} \mathcal{Q}=\mathcal{O}_{M}\left(P+D_{Q}\right) \otimes\left(\mathcal{Q} / \mathcal{O}_{M}\left(P+D_{Q}\right)\right) .
$$

So, $\mathcal{Q} / \mathcal{O}_{M}\left(P+D_{Q}\right)=\mathcal{O}_{M}\left(-D_{R}\right)$. Consequently, from (4.13), we get a short exact sequence of vector bundles

$$
0 \longrightarrow \mathcal{O}_{M}\left(P+D_{Q}\right) \longrightarrow \mathcal{Q} \longrightarrow \mathcal{O}_{M}\left(-D_{R}\right) \longrightarrow 0
$$
Let

To complete the proof of the lemma, we need to show that the short exact sequence in (4.14) does not split.

$$
\omega \in H^{1}\left(M, \operatorname{Hom}\left(\mathcal{O}_{M}\left(-D_{R}\right), \mathcal{O}_{M}\left(P+D_{Q}\right)\right)=H^{1}\left(M, \mathcal{O}_{M}\left(P+D_{Q}+D_{R}\right)\right)\right.
$$

be the extension class for the exact sequence in (4.14). We will now compute $\omega$.

From (4.6) and (4.7) we have the short exact sequence

$$
0 \longrightarrow \mathcal{O}_{M}(P) \oplus \mathcal{O}_{M}\left(D_{Q}\right) \longrightarrow E \longrightarrow \mathcal{O}_{M}\left(-D_{R}\right) \longrightarrow 0
$$

of holomorphic vector bundles on $M$. Let

$$
\begin{aligned}
\theta^{\prime} \in & H^{1}\left(M,\left(\mathcal{O}_{M}(P) \oplus \mathcal{O}_{M}\left(D_{Q}\right)\right) \otimes \mathcal{O}_{M}\left(D_{R}\right)\right) \\
& =H^{1}\left(M, \mathcal{O}_{M}\left(P+D_{R}\right)\right) \oplus H^{1}\left(M, \mathcal{O}_{M}\left(D_{Q}+D_{R}\right)\right)
\end{aligned}
$$

be the cohomology class for this exact sequence. Evidently, $\theta^{\prime}$ coincides with

$$
(0, \theta) \in H^{1}\left(M, \mathcal{O}_{M}\left(P+D_{R}\right)\right) \oplus H^{1}\left(M, \mathcal{O}_{M}\left(D_{Q}+D_{R}\right)\right),
$$

where $\theta$ is the class in (4.5).

Next, consider the homomorphism $\gamma$ defined by the composition

$$
\left.\mathcal{O}_{M}\left(D_{Q}\right) \hookrightarrow \mathcal{O}_{M}(P) \oplus \mathcal{O}_{M}\left(D_{Q}\right) \rightarrow\left(\mathcal{O}_{M}(P) \oplus \mathcal{O}_{M}\left(D_{Q}\right)\right) / \sigma_{1}(F)=\mathcal{O}_{M}\left(P+D_{Q}\right)\right)
$$

(see (4.12)), where the homomorphism

$$
\mathcal{O}_{M}\left(D_{Q}\right) \hookrightarrow \mathcal{O}_{M}(P) \oplus \mathcal{O}_{M}\left(D_{Q}\right)
$$


is the inclusion of the second factor. Clearly, this composition $\gamma$ coincides with the natural inclusion of the coherent sheaf $\mathcal{O}_{M}\left(D_{Q}\right)$ in $\mathcal{O}_{M}\left(P+D_{Q}\right)$ ). Therefore, the cohomology classes $\omega$ and $\theta$ (constructed in (4.15) and (4.5)) satisfy the equation

$$
\omega=\rho(\theta)
$$

where

$$
\rho: H^{1}\left(M, \mathcal{O}_{M}\left(D_{Q}+D_{R}\right)\right) \longrightarrow H^{1}\left(M, \mathcal{O}_{M}\left(P+D_{Q}+D_{R}\right)\right)
$$

is the homomorphism induced by the natural inclusion map

$$
\mathcal{O}_{M}\left(D_{Q}+D_{R}\right) \hookrightarrow \mathcal{O}_{M}\left(P+D_{Q}+D_{R}\right)
$$

of coherent analytic sheaves. Consider the short exact sequence of coherent sheaves

$$
0 \longrightarrow \mathcal{O}_{M}\left(D_{Q}+D_{R}\right) \longrightarrow \mathcal{O}_{M}\left(P+D_{Q}+D_{R}\right) \longrightarrow \mathcal{O}_{M}\left(P+D_{Q}+D_{R}\right)_{P} \longrightarrow 0,
$$

where $\mathcal{O}_{M}\left(P+D_{Q}+D_{R}\right)_{P}$ is the torsion sheaf supported at $P$ with its stalk being the fiber of the line bundle $\mathcal{O}_{M}\left(P+D_{Q}+D_{R}\right)$ over $P$. Let

$$
\begin{aligned}
0 & \longrightarrow H^{0}\left(M, \mathcal{O}_{M}\left(D_{Q}+D_{R}\right)\right) \longrightarrow H^{0}\left(M, \mathcal{O}_{M}\left(P+D_{Q}+D_{R}\right)\right) \\
& \stackrel{\alpha_{1}}{\longrightarrow} \mathcal{O}_{M}\left(P+D_{Q}+D_{R}\right)_{P} \stackrel{\alpha_{2}}{\longrightarrow} H^{1}\left(M, \mathcal{O}_{M}\left(D_{Q}+D_{R}\right)\right) \\
& \stackrel{\rho}{\longrightarrow} H^{1}\left(M, \mathcal{O}_{M}\left(P+D_{Q}+D_{R}\right)\right)
\end{aligned}
$$

be the long exact sequence of cohomologies associated with it. We have

$$
\operatorname{dim} H^{0}\left(M, \mathcal{O}_{M}\left(P+D_{Q}+D_{R}\right)\right)=\operatorname{dim} H^{0}\left(M, \mathcal{O}_{M}\left(K_{M}\right)\right)=g
$$

and, by Riemann-Roch and (4.4),

$$
\operatorname{dim} H^{0}\left(M, \mathcal{O}_{M}\left(D_{Q}+D_{R}\right)\right)=g-1 .
$$

These imply that $\alpha_{1}$ in (4.17) is surjective. Therefore, $\alpha_{2}$ in (4.17) is the zero homomorphism. This implies that $\rho$ in (4.17) is injective.

Since $\rho$ is injective, from (4.16) it follows that $\omega \neq 0$, because $\theta \neq 0$ (see (4.5)). The exact sequence in (4.14) does not split because $\omega \neq 0$.

Proposition 4.2 The holomorphic vector bundle $\mathcal{Q}$ in (4.14) admits a holomorphic connection.

Proof Assume that $\mathcal{Q}$ does not admit any holomorphic connection. Since $\operatorname{degree}(\mathcal{Q})=0$, and $\mathcal{Q}$ does not admit any holomorphic connection, the criterion of Atiyah-Weil says that $\mathcal{Q}$ holomorphically decomposes as

$$
\mathcal{Q}=L \oplus \mathcal{M}
$$

where degree $(L)=-\operatorname{degree}(\mathcal{M})>0$. Let $p_{\mathcal{M}}: \mathcal{Q} \longrightarrow \mathcal{M}$ be the projection given by the decomposition in (4.18). Let $\beta$ denote the composition

$$
\mathcal{O}_{M}\left(P+D_{Q}\right) \hookrightarrow \mathcal{Q} \stackrel{p_{\mathcal{M}}}{\longrightarrow} \mathcal{M},
$$

where the inclusion is constructed in (4.13). Since

$$
\operatorname{degree}\left(\mathcal{O}_{M}\left(P+D_{Q}\right)\right)=g-1>0>\operatorname{degree}(\mathcal{M}),
$$

there is no nonzero homomorphism from $\mathcal{O}_{M}\left(P+D_{Q}\right)$ to $\mathcal{M}$. In particular, $\beta=0$.

We have $\mathcal{O}_{M}\left(P+D_{Q}\right) \subset L$ because $\beta=0$. Since both $\mathcal{O}_{M}\left(P+D_{Q}\right)$ and $L$ are line subbundles on $\mathcal{Q}$, this implies that the two subbundles $\mathcal{O}_{M}\left(P+D_{Q}\right)$ and $L$ coincide. Hence,

$$
\mathcal{M}=\mathcal{Q} / L=\mathcal{Q} / \mathcal{O}_{M}\left(P+D_{Q}\right)=\mathcal{O}_{M}\left(-D_{R}\right)
$$

(see Lemma 4.1). Therefore, the decomposition $\mathcal{Q}=L \oplus \mathcal{M}$ in (4.18) produces a splitting of the short exact sequence in (4.14). But we know from Lemma 4.1 that the short exact sequence in (4.14) does not split. In view of the above contradiction, we conclude that $\mathcal{Q}$ admits a holomorphic connection.

As we have seen, $E$ does not admit a holomorphic connection. On the other hand, consider the short exact sequence in (4.11). The trivial holomorphic line bundle $F=\mathcal{O}_{M}$ admits the trivial holomorphic connection. The quotient bundle $\mathcal{Q}$ is flat by Proposition 4.2.

Therefore, the question in Sect. 3.1 has a negative answer. 


\section{Logarithmic connections on a Riemann surface}

The multiplicative group $\mathbb{C} \backslash\{0\}$ will be denoted by $\mathbb{G}_{m}$. A torus is a product of copies of $\mathbb{G}_{m}$. Any two maximal tori in a complex algebraic group are conjugate [8, p. 158, Proposition 11.23(ii)].

By a homomorphism between algebraic groups or by a character we will always mean a holomorphic homomorphism or a holomorphic character.

Let $M$ be a compact connected Riemann surface. Fix a finite subset

$$
D:=\left\{x_{1}, \ldots, x_{n}\right\} \subset M .
$$

The reduced effective divisor $x_{1}+\cdots+x_{n}$ will also be denoted by $D$. The subsheaf $T M \otimes \mathcal{O}_{M}(-D)$ of $T M$ will be denoted by $T M(-D)$.

Let $G$ be a complex Lie group.

$$
f: E_{G} \longrightarrow M
$$

be a holomorphic principal $G$-bundle over $M$. Consider the Atiyah exact sequence in (2.4). Now define

$$
\operatorname{At}\left(E_{G}, D\right):=\left(d^{\prime} f\right)^{-1}(T M(-D)) \subset \operatorname{At}\left(E_{G}\right),
$$

where $d^{\prime} f$ is the projection in (2.4). So from (2.4) we have the exact sequence of vector bundles on $M$

$$
0 \longrightarrow \operatorname{ad}\left(E_{G}\right) \stackrel{i_{0}}{\longrightarrow} \operatorname{At}\left(E_{G}, D\right) \stackrel{\sigma}{\longrightarrow} T M(-D) \longrightarrow 0,
$$

where $\sigma$ is the restriction of $d^{\prime} f$ to $\operatorname{At}\left(E_{G}, D\right) \subset \operatorname{At}\left(E_{G}\right)$. The exact sequence in (5.1) will be called the logarithmic Atiyah exact sequence for $E_{G}$.

A logarithmic connection on $E_{G}$ singular over $D$ is a holomorphic homomorphism

$$
\theta: T M(-D) \longrightarrow \operatorname{At}\left(E_{G}, D\right)
$$

such that $\sigma \circ \theta=\operatorname{Id}_{T M(-D)}$, where $\sigma$ is the homomorphism in (5.1). Note that giving such a homomorphism $\theta$ is equivalent to giving a homomorphism

$$
\varpi: \operatorname{At}\left(E_{G}, D\right) \longrightarrow \operatorname{ad}\left(E_{G}\right)
$$

such that $\varpi \circ i_{0}=\operatorname{Id}_{\mathrm{ad}\left(E_{H}\right)}$, where $i_{0}$ is the homomorphism in (5.1).

So, a holomorphic principal $G$-bundle admits a logarithmic connection singular over $D$ if and only if the logarithmic Atiyah exact sequence in (5.1) splits holomorphically.

\subsection{Residue of a logarithmic connection}

Given a vector bundle $W$ on $M$, the fiber of $W$ over any point $x \in M$ will be denoted by $W_{x}$. For any $\mathcal{O}_{M}$-linear homomorphism $f: W \longrightarrow V$ of holomorphic vector bundles, its restriction $W_{x} \longrightarrow V_{x}$ will be denoted by $f(x)$.

From (2.4) and (5.1), we have the commutative diagram of homomorphisms

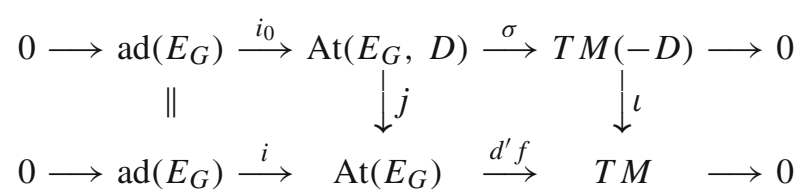

on $M$. So for any point $x \in M$, we have

$$
\mathrm{d}^{\prime} f(x) \circ j(x)=\iota(x) \circ \sigma(x): \operatorname{At}\left(E_{G}, D\right)_{x} \longrightarrow(T M)_{x}=T_{x} M .
$$

Note that $\iota(x)=0$ if $x \in D$; therefore, in that case $\mathrm{d}^{\prime} f(x) \circ j(x)=0$. Consequently, for every $x \in D$ there is a homomorphism

$$
R_{x}: \operatorname{At}\left(E_{G}, D\right)_{x} \longrightarrow \operatorname{ad}\left(E_{G}\right)_{x}
$$


uniquely defined by the identity $i(x) \circ R_{x}(v)=j(x)(v)$ for all $v \in \operatorname{At}\left(E_{G}, D\right)_{x}$. Note that

$$
R_{x} \circ i_{0}(x)=\operatorname{Id}_{\mathrm{ad}\left(E_{G}\right)_{x}},
$$

where $i_{0}$ is the homomorphism in (5.3). Therefore, from (5.1), we have

$$
\operatorname{At}\left(E_{G}, D\right)_{x}=\operatorname{ad}\left(E_{G}\right)_{x} \oplus \operatorname{kernel}\left(R_{x}\right)=\operatorname{ad}\left(E_{G}\right)_{x} \oplus T M(-D)_{x} ;
$$

note that the composition of homomorphisms

$$
\operatorname{kernel}\left(R_{x}\right) \hookrightarrow \operatorname{At}\left(E_{G}, D\right)_{x} \stackrel{\sigma(x)}{\longrightarrow} T M(-D)_{x}
$$

is an isomorphism.

For any $x \in D$, the fiber $T M(-D)_{x}$ is identified with $\mathbb{C}$ using the Poincare adjunction formula [12, p. 146]. Indeed, for any holomorphic coordinate $z$ around $x$ with $z(x)=0$, the image of $z \frac{\partial}{\partial z}$ in $T M(-D)_{x}$ is independent of the choice of the coordinate function $z$; the above-mentioned identification between $T M(-D)_{x}$ and $\mathbb{C}$ sends this independent image to $1 \in \mathbb{C}$. Therefore, from (5.5), we have

$$
\operatorname{At}\left(E_{G}, D\right)_{x}=\operatorname{ad}\left(E_{G}\right)_{x} \oplus \mathbb{C}
$$

for all $x \in D$.

For a logarithmic connection $\theta: T M(-D) \longrightarrow \operatorname{At}\left(E_{G}, D\right)$ as in (5.2), and any $x \in D$, define

$$
\operatorname{Res}(\theta, x):=R_{x}(\theta(1)) \in \operatorname{ad}\left(E_{G}\right)_{x},
$$

where $R_{x}$ is the homomorphism in (5.4); in the above definition 1 is considered as an element of $T M(-D)_{x}$ using the identification of $\mathbb{C}$ with $T M(-D)_{x}$ mentioned earlier.

The element $\operatorname{Res}(\theta, x)$ in (5.7) is called the residue, at $x$, of the logarithmic connection $\theta$.

Let $S$ be a complex affine algebraic group and

$$
\rho: H \longrightarrow S
$$

a homomorphism. As before, $E_{G}$ is a holomorphic principal $G$-bundle on $M$. Let

$$
E_{S}:=E_{G} \times{ }^{\rho} S \longrightarrow M
$$

be the holomorphic principal $S$-bundle obtained by extending the structure group of $E_{G}$ using $\rho$. So $E_{S}$ is the quotient of $E_{G} \times S$ obtained by identifying $(y, m)$ and $\left(y h^{-1}, \rho(h) m\right)$, where $y, m$ and $h$ run over $E_{G}, S$ and $G$, respectively. Therefore, we have a morphism

$$
\widehat{\rho}: E_{G} \longrightarrow E_{S}, \quad y \longmapsto \widetilde{\left(y, e_{S}\right)},
$$

where $\widetilde{\left(y, e_{S}\right)}$ is the equivalence class of $\left(y, e_{S}\right)$ with $e_{S}$ being the identity element of $S$. The homomorphism of Lie algebras $d \rho: \mathfrak{g} \longrightarrow \mathfrak{s}:=\operatorname{Lie}(S)$ associated with $\rho$ produces a homomorphism of vector bundles

$$
\alpha: \operatorname{ad}\left(E_{G}\right) \longrightarrow \operatorname{ad}\left(E_{S}\right) .
$$

The maps $\widehat{\rho}$ and $d \rho$ together produce a homomorphism of vector bundles

$$
\widetilde{A}: \operatorname{At}\left(E_{G}\right) \longrightarrow \operatorname{At}\left(E_{S}\right) \text {. }
$$

This map $\widetilde{A}$ produces a homomorphism

$$
A: \operatorname{At}\left(E_{G}, D\right) \longrightarrow \operatorname{At}\left(E_{S}, D\right),
$$

which fits in the following commutative diagram of homomorphisms

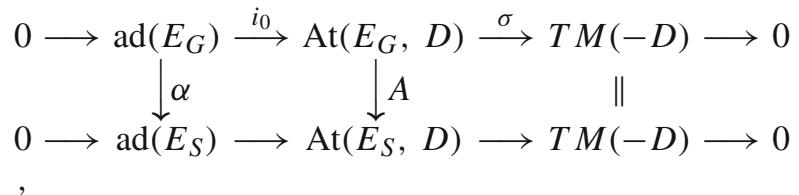


where the top exact sequence is the one in (5.1) and the bottom one is the corresponding sequence for $E_{S}$.

If $\theta: T M(-D) \longrightarrow \operatorname{At}\left(E_{G}, D\right)$ is a logarithmic connection on $E_{G}$ as in (5.2), then

$$
A \circ \theta: T M(-D) \longrightarrow \operatorname{At}\left(E_{S}, D\right)
$$

is a logarithmic connection on $E_{S}$ singular over $D$. From the definition of residue in (5.7) it follows immediately that

$$
\alpha(\operatorname{Res}(\theta, x))=\operatorname{Res}(A \circ \theta, x)
$$

for all $x \in D$. This proves the following:

Lemma 5.1 With the above notation, if $E_{G}$ admits a logarithmic connection $\theta$ singular over $D$ with residue $w_{x} \in \operatorname{ad}\left(E_{G}\right)_{x}$ at each $x \in D$, then $E_{S}$ admits a logarithmic connection $\theta^{\prime}=A \circ \theta$ singular over $D$ with residue $\alpha\left(w_{x}\right)$ at each $x \in D$.

Now we set $G=\operatorname{GL}(r, \mathbb{C})$.

Take a holomorphic vector bundle $E$ on $M$. The group of all holomorphic automorphisms of $E$ will be denoted by $\operatorname{Aut}(E)$. Let

$$
T \subset \operatorname{End}\left(E_{x}\right)
$$

be an endomorphism such that $\phi(x) \circ T=T \circ \phi(x)$ for all $\phi \in \operatorname{Aut}(E)$.

Lemma 5.2 Let $V \subset E$ be a holomorphic subbundle which is a holomorphic direct summand. Then $T\left(V_{x}\right) \subset$ $V_{x}$.

Proof Take a holomorphic subbundle $W \subset E$ such that $E=V \bigoplus W$. For nonzero complex numbers $a, b$, we have the automorphism $A(a, b) \in \operatorname{Aut}(E)$ which acts on $V$ (respectively, $W$ ) as multiplication by $a$ (respectively, $b)$. Since $T$ commutes with $A(a, b)(x)$ for all $a, b \in \mathbb{C} \backslash\{0\}$, it follows that $T\left(V_{x}\right) \subset V_{x}$.

Take a holomorphic vector bundle $E$ on $M$. Fix a finite subset

$$
D:=\left\{x_{1}, \ldots, x_{n}\right\} \subset M .
$$

For each point $x \in D$, fix

$$
T_{x} \in \operatorname{End}\left(E_{x}\right)
$$

such that $\phi(x) \circ T_{x}=T_{x} \circ \phi(x)$ for all $\phi \in \operatorname{Aut}(E)$.

The following theorem is proved in [4]:

Theorem 5.3 There is a logarithmic connection $\theta$ on $E$ singular over $D$ such that

$$
\operatorname{Res}(\theta, x)=T_{x}
$$

if and only if

$$
\operatorname{degree}(V)+\sum_{x \in D} \operatorname{trace}\left(\left.\operatorname{Res}(\theta, x)\right|_{V_{x}}\right)=0 .
$$
3.

Note that when $D=0$, then Theorem 5.3 reduces to the criterion of Atiyah and Weil mentioned in Sect.

Acknowledgements We thank the two referees for going through the paper carefully. The second-named author is partially supported by a J. C. Bose Fellowship.

Open Access This article is licensed under a Creative Commons Attribution 4.0 International License, which permits use, sharing, adaptation, distribution and reproduction in any medium or format, as long as you give appropriate credit to the original author(s) and the source, provide a link to the Creative Commons licence, and indicate if changes were made. The images or other third party material in this article are included in the article's Creative Commons licence, unless indicated otherwise in a credit line to the material. If material is not included in the article's Creative Commons licence and your intended use is not permitted by statutory regulation or exceeds the permitted use, you will need to obtain permission directly from the copyright holder. To view a copy of this licence, visit http://creativecommons.org/licenses/by/4.0/. 


\section{References}

1. Atiyah, M.F.: On the Krull-Schmidt theorem with application to sheaves. Bull. Soc. Math. Fr. 84, 307-317 (1956)

2. Atiyah, M.F.: Complex analytic connections in fibre bundles. Trans. Am. Math. Soc. 85, 181-207 (1957)

3. Azad, H.; Biswas, I.: On holomorphic principal bundles over a compact Riemann surface admitting a flat connection. Math. Ann. 322, 333-346 (2002)

4. Biswas, I.; Dan, A.; Paul, A.: Criterion for logarithmic connections with prescribed residues. Manuscripta Math. 155, 77-88 (2018)

5. Biswas, I.; Dumitrescu, S.: Branched holomorphic Cartan geometries and Calabi-Yau manifolds. Int. Math. Res. Not. 74287458, 2019

6. Biswas, I.; Heu, V.: Non-flat extension of flat vector bundles, Int. J. Math. 26 (2015), no. 14, 1550114.

7. Biswas, I.; Subramanian, S.: Flat holomorphic connections on principal bundles over a projective manifold. Trans. Am. Math. Soc. 356, 3995-4018 (2004)

8. Borel, A.: Linear algebraic groups. Second edition, Graduate Texts in Mathematics, 126. Springer-Verlag, New York, 1991.

9. Druel, S.: A decomposition theorem for singular spaces with trivial canonical class of dimension at most five. Invent. Math. 211, 245-296 (2018)

10. Ehresmann, C.: Sur les espaces localement homogènes. L’Enseign. Math. 35, 317-333 (1936)

11. Fassarella, T.; Loray, F.: Flat parabolic vector bundles on elliptic curves. J. Reine Angew. Math. 761, 81-122 (2020)

12. Griffiths, P.; Harris, J.: Principles of algebraic geometry. Pure and Applied Mathematics. Wiley-Interscience, New York (1978)

13. Humphreys, J. E.: Linear algebraic groups. Graduate Texts in Mathematics, No. 21, Springer-Verlag, New York-Heidelberg, 1975

14. Jahnke, P.; Radloff, I.: Projective uniformization, extremal Chern classes and quaternionic Shimura curves. Math. Ann. 363, 753-776 (2015)

15. Kobayashi, S.; Nomizu, K.: Foundations of differential geometry. Vol I, Interscience Publishers, a division of John Wiley \& Sons, New York-London, 1963

16. Simpson, C.T.: Higgs bundles and local systems. Inst. Hautes Études Sci. Publ. Math. 75, 5-95 (1992)

17. Weil, A.: Généralisation des fonctions abéliennes. J. Math. Pures Appl. 17, 47-87 (1938)

Publisher's Note Springer Nature remains neutral with regard to jurisdictional claims in published maps and institutional affiliations. 